\title{
$6 \mathrm{MeV}$ heavy i on beam probe on the Lar ge Hel i cal Devi ce
}

\begin{tabular}{|l|l|}
\hline $\begin{array}{l}\text { j our nal or } \\
\text { publ i cat i on t i t l e }\end{array}$ & Revi ew of Sci ent i f i c I nst r ument s \\
\hline vol une & Vol . 77 \\
\hline number & I ssue10 \\
\hline page r ange & pp. 10F523 1 10F523- 4 \\
\hline year & 2006- 10-01 \\
\hline URL & ht t p: //hdl . handl e. net /10655/3776 \\
\hline
\end{tabular}




\title{
$6 \mathrm{MeV}$ heavy ion beam probe on the Large Helical Device
}

\author{
T. Ido, ${ }^{\text {a) }}$ A. Shimizu, M. Nishiura, A. Nishizawa, ${ }^{\text {b) }}$ S. Katoh, K. Tsukada, M. Yokota, \\ H. Ogawa, T. Inoue, Y. Hamada, and LHD Experimental Group \\ National Institute for Fusion Science, Oroshi-cho, Toki-shi, Gifu 509-5292, Japan \\ T. P. (Crowley ${ }^{\text {c) }}$ \\ Rensselaer Polytechnic Institute, 110 8th Street, Troy, New York 12180
}

(Received 8 May 2006; presented on 11 May 2006; accepted 25 July 2006; published online 9 October 2006)

\begin{abstract}
A heavy ion beam probe (HIBP) has been installed on the Large Helical Device (LHD). A $\mathrm{MeV}$-range beam is required for the LHD-HIBP. The probing beam is accelerated up to $6 \mathrm{MeV}$ by use of a tandem accelerator. A new energy analyzer with tandem electrodes has also been developed to analyze such a high energy beam. As a result, a secondary beam can be detected and its energy successfully analyzed. It is verified, in principle, that the potential profile can be measured using the HIBP. ㅇ 2006 American Institute of Physics. [DOI: 10.1063/1.2338311]
\end{abstract}

\section{INTRODUCTION}

The radial electric field in magnetically confined nonaxisymmetric plasmas has been studied because it is an important parameter in neoclassical plasma transport. In addition, since the discovery of the $H$ mode, ${ }^{1}$ the suppression of plasma turbulence by shear flow induced by the gradient of the radial electric field ${ }^{2}$ has been a point of focus of the study of magnetically confined plasma. Therefore, measurement of the radial electric field is important for transport study.

Heavy ion beam probes ${ }^{3}$ (HIBPs) have been applied in various magnetically confined plasmas in order to measure the electrostatic potential, its fluctuation, and density fluctuation at the same position simultaneously. So far, many important results regarding plasma transport have been obtained through the use of HIBPs. We have developed an HIBP for the Large Helical Device (LHD) in order to measure its plasma potential profile. ${ }^{4,5}$ In this article, we will describe the HIBP apparatus and its expected performance.

\section{APPARATUS}

Figure 1 shows a schematic of the LHD-HIBP system. Singly charged positive ions, referred to as the primary beam, are injected from a lower vertical port of the LHD. They are ionized on their trajectory in the plasma due to collision with the plasma. Some of them become doubly charged ions, referred to as the secondary beam, and depart from the plasma. They are selected by slits in a horizontal port of the LHD shown in Fig. 1, and their energy is analyzed.

The beam line is composed of a negative ion source, a tandem accelerator, deflectors, electrostatic lenses, two octu-

\footnotetext{
${ }^{a)}$ Electronic mail: ido@LHD.nifs.ac.jp

${ }^{b)}$ Present address: Tono Geoscience Centre, Japan Atomic Energy Agency, Toki, Gifu, Japan.

${ }^{c}$ Present address: National Institute of Standards and Technology, Boulder, CO 80305.
}

pole sweepers, and a tandem-electrode energy analyzer. Each device is described in the following subsections.

\section{A. The tandem accelerator and negative ion source}

Since the magnetic field of the LHD is up to $3 \mathrm{~T}$ and the Larmor radius of the probing beam has to be a few meters in order to detect the secondary beam at the detector shown in Fig. 1, a MeV-range beam of heavy ions is required for the LHD-HIBP. In order to obtain such a high energy beam, a tandem accelerator is used. In the tandem accelerator, singly charged negative ions are initially accelerated toward a gas cell located at the center of the accelerator, where high positive voltage is applied. They are ionized positively by the collision with neutral particles in the gas cell, and are then accelerated again toward the output port which is grounded. Thus, the tandem accelerator can accelerate the beam twice, and the acceleration voltage is reduced to half of the required beam energy. In the LHD-HIBP, the acceleration voltage is up to $3 \mathrm{MV}$, so singly charged positive ions having energy of up to $6 \mathrm{MeV}$ are available.

For use of the tandem accelerator, a negative ion source is required as mentioned above. We selected a gold negative ion source because the ion's mass is suitable for the HIBP and some gold negative ion sources had been developed in other laboratories. Therefore, in the LHD-HIBP, singly charged positive ions $\left(\mathrm{Au}^{+}\right)$are injected, and doubly charged ions $\left(\mathrm{Au}^{2+}\right)$ ionized in the plasma are detected. We have developed negative gold ion sources whose maximum output current is $12 \mu \mathrm{A}$ in the steady operation. ${ }^{6}$

However, the tandem accelerator also has a disadvantage. The accelerated negative ions are ionized in the gas cell at the center of the accelerator, so the output current depends on the ionization rate. The current ratio of the singly charged ions to the incident negative ions is 0.1 or less. Thus, only a probe beam of $1 \mu \mathrm{A}$ is available, which is inadequate for measurement of plasma fluctuations. This requires future improvement of the negative ion source and the gas cell. 


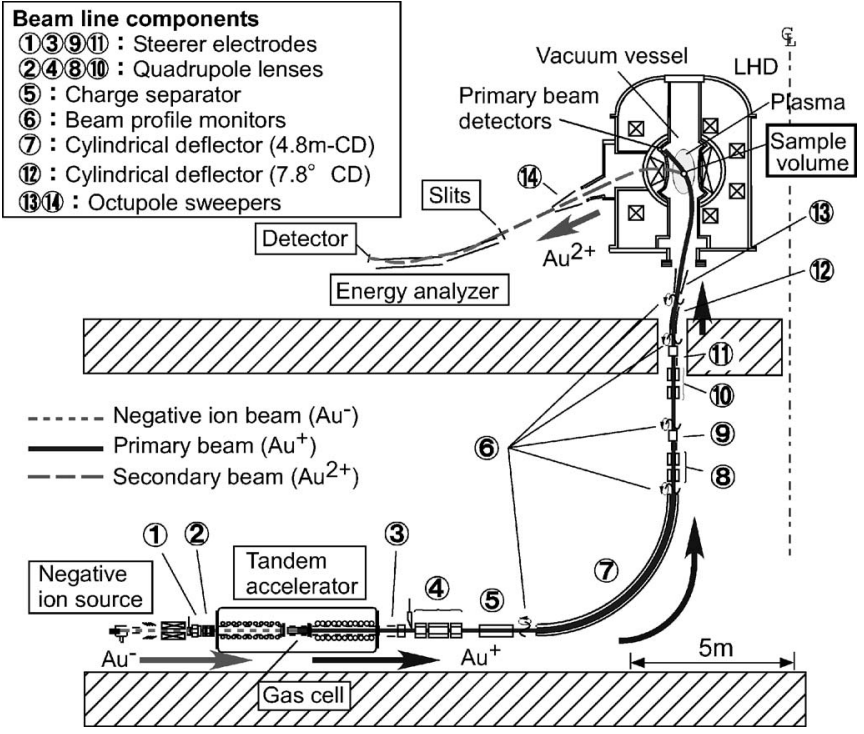

FIG. 1. Beam line of LHD-HIBP.

\section{B. Beam control devices (deflector, steering electrodes, and lenses)}

In order to transport the probe beam though the whole beam line with a total length of about $30 \mathrm{~m}$, some electrostatic devices are installed to control the beam trajectory and the focusing property.

As shown in Fig. 1, the ion source and the accelerator are installed horizontally in the basement of the LHD building in order to allow easy maintenance and to avoid the stray magnetic field of the LHD. The entrance port to the LHD is the lower-vertical port, so the beam extracted horizontally from the accelerator must be deflected at $90^{\circ}$ towards the LHD. For this purpose, an electrostatic cylindrical deflector with a radius of $4.8 \mathrm{~m}$ was installed (referred to as $4.8 \mathrm{~m}$ $\mathrm{CD}$ ). The singly charged ions with the energy of $6 \mathrm{MeV}$ can be deflected with the applied voltage of $37.5 \mathrm{kV}$ which is a practical voltage.

In order to adjust the focusing property, two electrostatic quadrupole lenses, referred to as a quadrupole doublet, are arranged as a focusing-defocusing pair. In the LHD-HIBP system, two doublets are installed downstream of the $4.8 \mathrm{~m}$ $\mathrm{CD}$ as shown by Nos. 8 and 10 in Fig. 1. The doublet shown by No. 8 in Fig. 1 compensates for the unfavorable focusing due to the $4.8 \mathrm{~m} \mathrm{CD}$, and that shown by No. 10 is used to optimize the size of sample volumes in the plasma which determines the spatial resolution.

Stray magnetic fields of the LHD cause deflection of the beam in the beam line. According to trajectory calculation, the deflection of a beam having an energy of $6 \mathrm{MeV}$ is $30 \mathrm{~mm}$ or more at the injection port when the LHD's magnetic field is $3 \mathrm{~T}$ on the magnetic axis, and the beam cannot go through the beam line because the radius of an aperture is $15 \mathrm{~mm}$. Steering electrodes are installed to compensate for the deflection, as shown by Nos. 9 and 11 in Fig. 1.

\section{Octupole sweepers}

In helical systems, the probing beam is deflected in the toroidal direction as well as in the radial direction, and the (a)

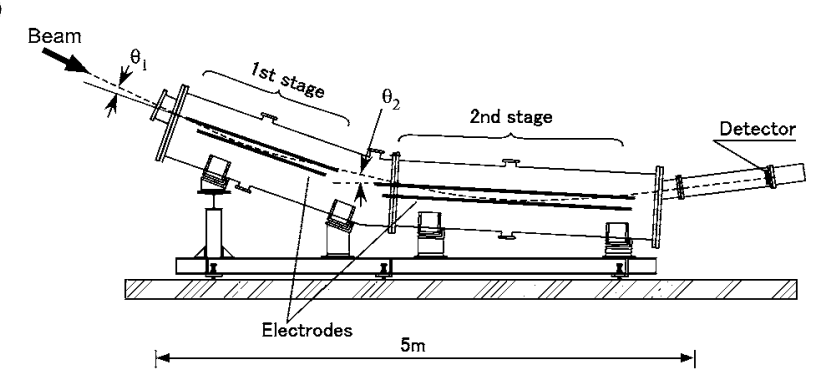

(b)

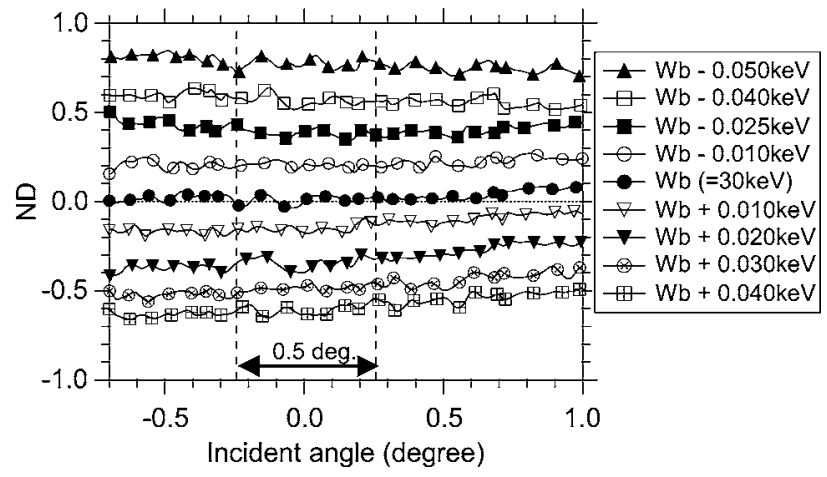

FIG. 2. (a) Tandem-electrode energy analyzer. $\theta_{1}=6^{\circ}$ and $\theta_{2}=10^{\circ}$. (b) The results of the calibration experiment using $30 \mathrm{keV}$ thallium beam. The horizontal axis is the change in the incident angle from the designed incident angle $\left(6^{\circ}\right)$. The vertical axis is the normalized position of the beam on the detector, and the change in the beam energy is estimated based on the change in the normalized position. Each marked line shows the incident angle dependence of the energy analysis of the beam whose energy values are shown in the legend, where $\mathrm{Wb}$ is $30 \mathrm{keV}$.

angle incident to the energy analyzer changes during the sweep of the probing beam as it measures the potential profile. This change in the incident angle causes error in the measurement of the beam energy because the energy is analyzed by measuring the range in the electric field of the energy analyzer. In the LHD-HIBP, two octupole sweepers are installed at the LHD's injection and extraction ports in order to apply the active trajectory control method. ${ }^{7}$ The sweeper at the injection port is used to select the position of the sample volume, while that at the extraction port is used to compensate for the change in the angle incident to the analyzer.

\section{Primary beam detectors}

Detectors with nine stainless steel plates were installed on the first wall of the LHD in order to detect the primary beam directly. The beam's trajectory and width are estimated by the use of the detectors.

\section{E. Tandem-electrodes energy analyzer}

The change in the energy of the extracted secondary beam is analyzed in order to measure plasma potential. So far, Green-Proca-type electrostatic analyzers ${ }^{8}$ have been used in HIBPs. However, this type of analyzer requires an impractical applied voltage $(\sim 400 \mathrm{kV})$ for $6 \mathrm{MeV}$ beams of the LHD-HIBP. Thus, we have devised a new energy analyzer with tandem electrodes, ${ }^{9}$ as shown in Fig. 2(a). Since the incident angles are $6^{\circ}$ and $10^{\circ}$ and are smaller than that of 


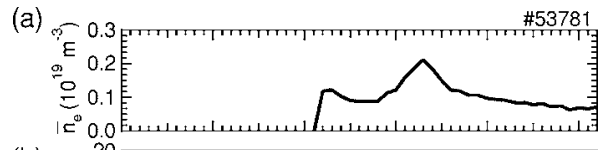

(b)

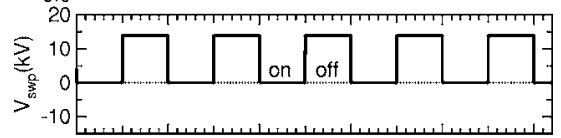

(c)

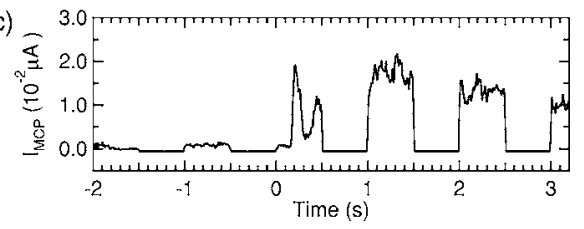

FIG. 3. (a) Line-averaged electron density. (b) Sweep voltage. (c) Detected secondary beam signal. The nominal gain of the MCP is $10^{5}$

the Green-Proca-type analyzer $\left(30^{\circ}\right)$, the voltages required to analyze the $6 \mathrm{MeV}$ beam decrease to 56.5 and $113.6 \mathrm{kV}$ for the first and second anodes, respectively.

The tandem-electrode energy analyzer has been calibrated by using $30 \mathrm{keV}$ thallium $\left(\mathrm{Tl}^{+}\right)$beam. The focusing property in relation to the incident angle $\left(\theta_{1}\right)$ is important for the HIBP. Figure 2(b) shows the position of the beam on the detector during the change in the incident angle, where the vertical axis is the position normalized by the beam size (ND). The change in the incident beam energy (Wb) is ideally proportional to that in ND. In Fig. 2(b), the position of the beam changes as the beam energy varies. This indicates that the beam energy can be analyzed. In addition, the dependence on the incident angle is weak near the designed incident angle. In the LHD-HIBP, the change in the incident angle is limited geometrically to within $0.5^{\circ}$ by an aperture and a slit. Therefore, the tandem analyzer is concluded to be available for the LHD-HIBP. According to the calibration experiment, the relation between the change in the normalized position ( $\Delta \mathrm{ND}$ ) and the change in the beam energy $(\Delta \mathrm{Wb})$ is $\Delta \mathrm{Wb}=-2.27 \times 10^{-3} \mathrm{~Wb} \times \Delta \mathrm{ND}$.

\section{F. Detector}

One of the most difficult problems for the LHD-HIBP is the attenuation of the probing beam current on its trajectory in plasmas. ${ }^{4}$ Thus, it is necessary that the small beam current be detected with high efficiency. We have developed a detector utilizing microchannel plates (MCPs). MCPs are used in beam probes in GAMMA-10 at Tsukuba University. ${ }^{10}$ However, they have not yet been used for MeV-range heavy ions. We examined the linearity of the detection efficiency of the MCP by use of singly charged gold ions $\left(\mathrm{Au}^{+}\right)$with the energy of 1-6 MeV. The results confirm that the MCP detector responds linearly to the incident beam current.

In addition, the MCPs respond to the radiation from the plasma as well as the probing ions. Thus, the performance of the MCPs should be examined in an actual experimental situation. The radiation from plasma does not produce a significant effect when the plasma density is low and the radiation power is small (as shown later in Fig. 3). Therefore, the MCPs are available as the detector of the LHD-HIBP.

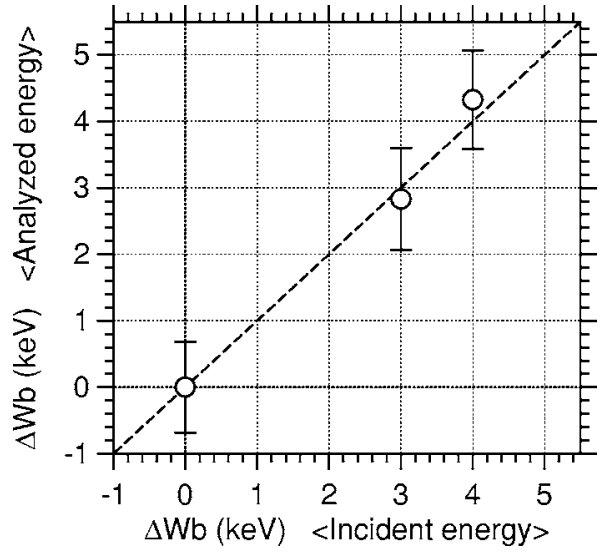

FIG. 4. Comparison between the incident and the analyzed beam energy. The horizontal and vertical axes are the incident and analyzed beam energies, respectively, and the energy is expressed as the difference from $5.042 \mathrm{MeV}$.

\section{PERFORMANCE AS A DIAGNOSTICS SYSTEM OF PLASMA POTENTIAL}

Experiments to detect the secondary beam were initially performed. Magnetic field strength of $2.75 \mathrm{~T}, 3.6 \mathrm{~m}$ major radius of the magnetic axis, and plasma is produced by electron cyclotron heating $(\mathrm{ECH})$. The incident energy of the probing beam of the HIBP is adjusted to $5.04 \mathrm{MeV}$ in order to observe the area near the center region of the plasma. The incident primary beam current is about $0.1 \mu \mathrm{A}$. Figure 3(a) shows the temporal behavior of the line-averaged electron density. Figure 3(b) shows the voltage of the injection-side sweeper. The primary beam is injected toward the center of the plasma while the sweep voltage is $0 \mathrm{kV}$ and the sample volume is located near the magnetic axis. On the other hand, the probing beam is blocked by the vacuum vessel of the LHD when the sweep voltage is $14 \mathrm{kV}$. The secondary beam signal is shown in Fig. 3(c), where the nominal current gain of the MCP is $10^{5}$. The signal is averaged for $10 \mathrm{~ms}$ because the secondary beam current is extremely small. It is detected only while the primary beam is injected towards the plasma. Therefore, the signal is not noise such as the radiation from the plasma, and the secondary beam produced in the plasma is detected successfully.

The secondary beam is also detected before the start of the discharge as shown in Fig. 3(c). It is produced through ionization by the neutral gas in the vacuum vessel; this process is referred to as gas ionization. Since there is no electric field in the vacuum vessel when it does not contain plasma, the energy of the secondary beam is identical to the incident energy of the primary beam. Thus, the secondary beam energy can be controlled by the adjustment of the primary beam energy, and in situ calibration of the energy analyzer can be performed by using the secondary beam produced through gas ionization. Figure 4 shows the analyzed secondary beam energies when energy values of the primary beam are $5.042,5.045(+3 \mathrm{kV})$, and $5.046 \mathrm{MeV}(+4 \mathrm{kV})$, respectively. The beam energy is analyzed successfully. The error bars come from the signal to noise ratio which is larger than the predicted plasma potential. However, the reason is that the secondary beam current produced by the gas ionization is 
quite small, as shown in Fig. 3(c), and the error bar can decrease to $100 \mathrm{eV}$ or less in the low density plasma $[\leqslant 1$ $\times 10^{19} \mathrm{~m}^{-3}$ ] because the secondary beam current is at least ten times larger than that in the gas-ionization experiment. Therefore, the change in the potential during the formation of the internal transport barrier, where the potential is predicted to change by a few hundreds volts, can be probably measured.

The development of high-power ion sources and efficient detectors to improve both the $\mathrm{S} / \mathrm{N}$ ratio and the temporal resolution constitute important subjects for future study.

\section{SUMMARY}

The MeV-range HIBP system has been installed on the LHD. The beam line works as designed, and the secondary beam produced in the plasma was successfully detected. The system can analyze the energy of the secondary beam. Therefore, it was verified, in principle, that the potential profile can be measured by the HIBP.

\section{ACKNOWLEDGMENTS}

The authors are grateful to Dr. H. Iguchi and Dr. A. Fujisawa of NIFS, Professor M. Sasao of Tohoku University,
Professor K. Ishii of Tsukuba University, and Professor M. Wada of Doshisha University for their fruitful discussions. Two of the authors (T.I.) and (A.N.) would like to thank Dr. A. Takagi and Dr. K. Ikegami of KEK for their valuable information regarding the development of negative ion sources. This work was supported by MEXT Japan under Grants-in-Aid for Scientific Research (Nos. 16760674 and 18760640) and the NIFS budget (Nos. NIFS05ULBB505, 507, and 508).

${ }^{1}$ F. Wagner et al., Phys. Rev. Lett. 49, 1408 (1989).

${ }^{2}$ H. Biglari, P. H. Diamond, and P. W. Terry, Phys. Fluids B 2, 1 (1990).

${ }^{3}$ R. L. Hickok, Rev. Sci. Instrum. 38, 142 (1967).

${ }^{4}$ A. Fujisawa, H. Iguchi, A. Taniike, M. Sasao, and Y. Hamada, IEEE Trans. Plasma Sci. 22, 395 (1994).

${ }^{5}$ A. Nishizawa et al., Proc. 10th International Conference and School on Plasma Physics and Controlled Fusion, Alushta, Ukraine, 10-20 Sept. 2004.

${ }^{6}$ M. Nishiura et al., Rev. Sci. Instrum. 77, 03A537 (2006).

${ }^{7}$ A. Fujisawa, H. Iguchi, S. Lee, T. P. Crowley, Y. Hamada, S. Hidekuma, and M. Kojima, Rev. Sci. Instrum. 67, 3099 (1996).

${ }^{8}$ T. S. Green and G. A. Proca, Rev. Sci. Instrum. 41, 1409 (1970).

${ }^{9}$ Y. Hamada, A. Fujisawa, H. Iguchi, A. Nishizawa, and Y. Kawasumi, Rev. Sci. Instrum. 68, 2020 (1997).

${ }^{10}$ K. Ishii, M. Kotoku, T. Segawa, I. Katanuma, A. Mase, and S. Miyoshi, Rev. Sci. Instrum. 60, 3270 (1989). 